PROCEEDINGS OF THE

AMERICAN MATHEMATICAL SOCIETY

Volume 134, Number 11, November 2006, Pages 3257-3266

S 0002-9939(06)08528-5

Article electronically published on May 12, 2006

\title{
A SPECTRAL COUNTABILITY CONDITION FOR ALMOST AUTOMORPHY OF SOLUTIONS OF DIFFERENTIAL EQUATIONS
}

\author{
NGUYEN VAN MINH, TOSHIKI NAITO, AND GASTON NGUEREKATA \\ (Communicated by Carmen C. Chicone)
}

\begin{abstract}
We consider the almost automorphy of bounded mild solutions to equations of the form

$(*)$

$d x / d t=A(t) x+f(t)$

with (generally unbounded) $\tau$-periodic $A(\cdot)$ and almost automorphic $f(\cdot)$ in a Banach space $\mathbb{X}$. Under the assumption that $\mathbb{X}$ does not contain $c_{0}$, the part of the spectrum of the monodromy operator associated with the evolutionary process generated by $A(\cdot)$ on the unit circle is countable. We prove that every bounded mild solution of $(*)$ on the real line is almost automorphic.
\end{abstract}

\section{INTRODUCTION}

Let us consider equations of the form

$$
\frac{d x}{d t}=A(t) x+f(t)
$$

where $A(t)$ is a (generally unbounded) linear operator on a Banach space $\mathbb{X}$ which is periodic, and $f$ is an $\mathbb{X}$-valued almost automorphic function on $\mathbb{R}$. We are interested in conditions for which every bounded mild solution of this equation is almost automorphic.

It is well known (see for example [10, 1, 5, 16 and the references therein) that if for the $t$-independent operator $A$ that is the generator of a $C_{0}$-semigroup, its imaginary part of the spectrum is countable and $\mathbb{X}$ does not contain any subspace isomorphic to $c_{0}$, then every bounded and uniformly continuous mild solution of (1.1) is almost periodic. The concept of almost automorphic functions, which was introduced by Bochner as an extension of one of the almost periodic functions, has recently caught the attention of many mathematicians (see for example [8, 13, 14, 15, 6, 11, 17, 4). It is natural to raise the question of whether this is true for almost automorphic solutions, that is, if $f$ is almost automorphic and $\mathbb{X}$ does not contain any subspace isomorphic to $c_{0}$, then is every bounded mild solution of (1.1) almost automorphic?

Received by the editors May 18, 2005.

2000 Mathematics Subject Classification. Primary 47D06; Secondary 34G10, 45M05.

Key words and phrases. Evolution equation, mild solution, almost automorphy, uniform spectrum.

(C)2006 American Mathematical Society 
In this paper we will prove that this is true. (In fact, we will prove a little more general assertion in which $A(t)$ generates a periodic evolutionary process.) As an almost automorphic function is not necessarily uniformly continuous, the methods of proving this standard result for almost periodic solutions, which are based on the uniform continuity of the bounded mild solutions and of the forcing term $f$, are no longer available. We refer the reader to 1, 10, for more details on the methods of proving this result. To overcome this difficulty, we will discretize the equations. We will first prove the discrete analog of the above problem, and then apply it to solve our main problem. In order to prove the discrete analog we will introduce a new concept of uniform spectrum of a bounded sequence with respect to a subspace that allows us to give a short proof of the claim for discrete equations. The main results of this paper are Theorems 2.14 and 3.2 .

\section{Almost automorphic functions And SEQUences}

\subsection{Almost automorphic functions.}

Definition 2.1. A function $f \in C(\mathbb{R}, \mathbb{X})$ is said to be almost automorphic if for any sequence of real numbers $\left(s_{n}^{\prime}\right)$, there exists a subsequence $\left(s_{n}\right)$ such that

$$
\lim _{m \rightarrow \infty} \lim _{n \rightarrow \infty} f\left(t+s_{n}-s_{m}\right)=f(t)
$$

for any $t \in \mathbb{R}$.

Theorem 2.2. Assume that $f, f_{1}$, and $f_{2}$ are almost automorphic and $\lambda$ is any scalar. Then the following hold true:

i) $\lambda f$ and $f_{1}+f_{2}$ are almost automorphic,

ii) $f_{\tau}(t):=f(t+\tau), t \in \mathbb{R}$, is almost automorphic,

iii) $\bar{f}(t):=f(-t), t \in \mathbb{R}$, is almost automorphic,

iv) the range $R_{f}$ of $f$ is precompact, so $f$ is bounded.

Proof. See [14, Theorems 2.1.3 and 2.1.4] for proofs.

Theorem 2.3. If $\left\{f_{n}\right\}$ is a sequence of almost automorphic $\mathbb{X}$-valued functions such that $f_{n} \mapsto f$ uniformly on $\mathbb{R}$, then $f$ is almost automorphic.

Proof. See [14, Theorem 2.1.10] for a proof.

If we equip $A A(\mathbb{X})$, the space of almost automorphic functions with the sup norm

$$
\|f\|_{\infty}=\sup _{t \in \mathbb{R}}\|f(t)\|
$$

then it turns out to be a Banach space. Recall that $A P(\mathbb{X})$ and $B C(\mathbb{R}, \mathbb{X})$ denote the spaces of all $\mathbb{X}$-valued almost periodic functions and all $\mathbb{X}$-valued bounded and continuous functions, respectively. The following inclusions are obvious:

$$
A P(\mathbb{X}) \subset A A(\mathbb{X}) \subset B C(\mathbb{R}, \mathbb{X}) .
$$

We refer the reader to [14 for information on the properties of the almost automorphic functions. In particular, we will use the following:

Theorem 2.4. Let us define $F: \mathbb{R} \mapsto \mathbb{X}$ by $F(t)=\int_{0}^{t} f(s) d s$, where $f \in A A(\mathbb{X})$. Then $F \in A A(\mathbb{X})$ iff $R_{F}=\{F(t) \mid t \in \mathbb{R}\}$ is precompact.

As a big difference between almost periodic functions and almost automorphic functions, we remark that an almost automorphic function is not necessarily uniform continuous, as shown in the following example due to B. M. Levitan. 
Example 2.5. The function

$$
f(t):=\sin \frac{1}{2+\cos t+\cos \sqrt{2} t}
$$

is almost automorphic, but not uniformly continuous. Therefore, it is not almost periodic.

2.2. Almost automorphic sequences. Similarly as for functions, we define below the almost automorphy of sequences. From now on we will use the notation $l^{\infty}(\mathbb{X})$ to indicate the space of all bounded (two-sided) sequences in a Banach space $\mathbb{X}$ with sup-norm, that is, if $x=\left\{x_{n}\right\}_{n \in \mathbb{Z}} \in l^{\infty}(\mathbb{X})$, then

$$
\|x\|:=\sup _{n \in \mathbb{Z}}\left\|x_{n}\right\| \text {. }
$$

Definition 2.6. A sequence $x \in l^{\infty}(\mathbb{X})$ ) is said to be almost automorphic if for any sequence of integers $\left(k_{n}^{\prime}\right)$, there exists a subsequence $\left(k_{n}\right)$ such that

$$
\lim _{m \rightarrow \infty} \lim _{n \rightarrow \infty} x_{p+k_{n}-k_{m}}=x_{p}
$$

for any $p \in \mathbb{Z}$.

The set of all almost automorphic sequences in $\mathbb{X}$ forms a closed subspace of $l^{\infty}(\mathbb{X})$ that is denoted by $a a(\mathbb{X})$. We can show that the range of an almost automorphic sequence is precompact. For each bounded sequence $g:=\left\{g_{n}\right\}_{n \in \mathbb{Z}}$ in $\mathbb{X}$, we will denote by $S(k) g$ the $k$-translation of $g$ in $l^{\infty}(\mathbb{X})$, i.e., $(S(k) g)_{n}=g_{n+k}, \forall n \in \mathbb{Z}$, and $S$ stands for $S(1)$.

2.3. Kadets Theorem. In this paper we will use the standard notation $c_{0}$ for the Banach space of all numerical sequences $\left\{a_{n}\right\}_{n=1}^{\infty}$ such that $\lim _{n \rightarrow \infty} a_{n}=0$, equipped with sup-norm. In the simplest case, the problem we are considering becomes the following: when is the integral of an almost automorphic function also almost automorphic? We can take the same counterexample as in [10] to show that additional conditions should be imposed on the space $\mathbb{X}$.

Example 2.7. Consider the function $f(t)$ with values in $c_{0}$ defined by

$$
f(t)=\{(1 / n) \cos (t / n)\}_{n=1}^{\infty}, \forall t \in \mathbb{R} .
$$

The integral $F(t)=\int_{0}^{t} f(\xi) d \xi$ of $f(t)$ is $F(t)=\{\sin (t / n)\}_{n=1}^{\infty}$. Obviously, $f$ is almost periodic (so it is almost automorphic), and $F$ is bounded. However, the range of $F$, as shown in [10, pp. 81-82], is not precompact, so $F$ cannot be almost automorphic.

The Kadets Theorem (see, e.g., [10, Theorem 2, p. 86]) says that if $f$ is almost periodic and $F$ is bounded, then $F$ is almost periodic if and only if $\mathbb{X}$ does not contain any subspace isomorphic to $c_{0}$. An extension of the Kadets Theorem to almost automorphic functions was given in [2].

The following extension of the Kadets Theorem to sequences will be used in this paper.

Lemma 2.8. Assume that $x=\left\{x_{n}\right\}_{n \in \mathbb{Z}}$ is a sequence in a Banach space $\mathbb{X}$ that does not contain any subspace isomorphic to $c_{0}$, and the difference

$$
x-S x=y
$$

is almost automorphic. Then, the sequence $x$ itself is almost automorphic. 
Proof. This lemma is a special case of [2, Theorem 1].

As is well known (see, e.g., [10]), a convex Banach space does not contain any subspace isomorphic to $c_{0}$. In particular, every finite-dimensional space does not contain any subspace isomorphic to $c_{0}$.

2.4. Spectral theory of bounded sequences. Below we denote by $S(n)$ the translation operator: $l^{\infty}(\mathbb{X}) \ni g . \mapsto g_{\cdot+n} \in l^{\infty}(\mathbb{X})$, where $n \in \mathbb{Z}$, and $S$ stands for $S(1)$.

Definition 2.9. Let $\mathcal{A}$ be a closed subspace of $l^{\infty}(\mathbb{X})$. We say that $\mathcal{A}$ satisfies Condition $H$ if the following conditions are satisfied:

i) Every sequence of the form (constant sequence) $\{a\}_{n \in \mathbb{Z}}$ is in $\mathcal{A}$.

ii) If $\left\{x_{n}\right\}_{n \in \mathbb{Z}} \in \mathcal{A}$ and $q \in \Gamma$, then the sequence $\left\{q^{n} x_{n}\right\}_{n \in \mathbb{Z}}$ is in $\mathcal{A}$.

iii) If $B$ is a bounded linear operator in $\mathbb{X}$, then $\left\{B x_{n}\right\}_{n \in \mathbb{Z}} \in \mathcal{A}$ whenever $\left\{x_{n}\right\}_{n \in \mathbb{Z}}$ is in $\mathcal{A}$.

iv) $S \mathcal{A}=\mathcal{A}$.

As an example of a subspace of $l^{\infty}(\mathbb{X})$ that satisfies Condition $\mathrm{H}$, we can take $a a(\mathbb{X})$.

In the rest of this paper we will always assume that $\mathcal{A}$ is a closed subspace of $l^{\infty}(\mathbb{X})$ that satisfies Condition $\mathrm{H}$. Then, in the quotient space $l^{\infty}(\mathbb{X}) / \mathcal{A}$ acts the operator $\tilde{S}$ defined by $\tilde{S}(x+\mathcal{A})=S x+\mathcal{A}, \forall x \in l^{\infty}(\mathbb{X})$. The operator $\tilde{S}$ is an isometric operator because of Condition $\mathrm{H}$ iv). Let $\pi$ be the canonical projection from $l^{\infty}(\mathbb{X})$ onto $l^{\infty}(\mathbb{X}) / \mathcal{A}$, and let us denote $\pi x=\tilde{x}$. Below, the notation $\mathcal{M}_{\hat{x}}$ means the closure of the subspace of $l^{\infty}(\mathbb{X}) / \mathcal{A}$ that spans over the set $\{\hat{S}(n) \hat{x}, n \in$ $\mathbb{Z}\}$.

Definition 2.10. The uniform spectrum of $x \in l^{\infty}(\mathbb{X})$ with respect to $\mathcal{A}$, which is denoted by $s p_{\mathcal{A}}(x)$, is defined to be the following:

$$
\sigma\left(\left.\tilde{S}\right|_{\mathcal{M}_{\tilde{x}}}\right) .
$$

It may be seen that $s p_{\mathcal{A}}(x)$ is part of the unit circle $\Gamma$.

Lemma 2.11. Let $x=\left\{x_{n}\right\}_{n \in \mathbb{Z}} \in l^{\infty}(\mathbb{X})$ and let $\mathcal{A}$ be a subspace of $l^{\infty}(\mathbb{X})$ that satisfies Condition H. Then $s p_{\mathcal{A}}(x)$ consists of all points $z_{0}$ of the unit circle $\Gamma$ such that the Carleman transform

$$
\hat{\tilde{x}}(\lambda):=\left\{\begin{array}{l}
\sum_{n=0}^{\infty} \lambda^{-n-1} \tilde{S}(n) \tilde{x}, \forall|\lambda|>1, \\
-\sum_{n=1}^{\infty} \lambda^{n-1} \tilde{S}(-n) \tilde{x}, \forall|\lambda|<1,
\end{array}\right.
$$

has no holomorphic extension to any neighborhood of $z_{0}$.

Proof. The proof follows the lines of the one in [12, Lemma 2.4], so we omit the details.

Consider the linear difference equation

$$
x_{n+1}=B x_{n}+f_{n}, \quad n \in \mathbb{Z},
$$

where $B$ is a bounded linear operator. Below we will denote by $\sigma_{\Gamma}(B)$ the part of the spectrum of $B$ on the unit circle $\Gamma$ of the complex plane. 
Lemma 2.12. Let $x \in l^{\infty}(\mathbb{X})$ be a solution of (2.4), and let $f \in \mathcal{A}$. Then

$$
s p_{\mathcal{A}}(x) \subset \sigma_{\Gamma}(B) .
$$

Proof. First we note that the operator of multiplication by $B$ in $l^{\infty}(\mathbb{X})$ has the same spectrum as $B$. For the reader's convenience we will use the same notation $B$ to indicate this multiplication operator if it does not cause any danger of confusion. So, by the definition of Condition $\mathrm{H}$, since the operator of multiplication by $B$ leaves $\mathcal{A}$ invariant, $B$ induces a bounded linear operator $\tilde{B}$ in the quotient space $l^{\infty}(\mathbb{X}) / \mathcal{A}$. Taking the Carleman transform of (2.4) we have

$$
\begin{aligned}
\widehat{\tilde{S}} \tilde{x}(\lambda) & =\tilde{B} \hat{\tilde{x}}(\lambda), \\
\lambda \hat{\tilde{x}}(\lambda)-\tilde{x} & =\tilde{B} \hat{\tilde{x}}(\lambda) .
\end{aligned}
$$

Therefore,

$$
\begin{aligned}
\lambda \hat{\tilde{x}}(\lambda)-\tilde{B} \hat{\tilde{x}}(\lambda) & =\tilde{x}, \\
(\lambda-\tilde{B}) \hat{\tilde{x}}(\lambda) & =\tilde{x} .
\end{aligned}
$$

Let $\eta_{0} \in \rho(\tilde{B})$. For $\eta$ close to $\eta_{0}$ such that $\eta \notin i \mathbb{R}$,

$$
\hat{\tilde{x}}(\eta)=(\eta-\tilde{B})^{-1} \tilde{x} .
$$

Using the analyticity of the resolvent $(\eta-\tilde{B})^{-1}$ with respect to $\eta$ in a neighborhood of $\eta_{0} \in \rho(B)$, we see that $\hat{\tilde{x}}(\eta)$ can be extended analytically to a neighborhood of $\eta_{0}$. Therefore, by Lemma 2.11, $s p_{\mathcal{A}}(x) \subset \sigma_{\Gamma}(\tilde{B}):=\Gamma \cap \sigma(\tilde{B})$. Now it suffices to show that $\sigma(\tilde{B}) \subset \sigma(B)$ to complete the proof. To this end, suppose that $z_{0} \in \rho(B)$; we will show that $z_{0}$ is in $\rho(\tilde{B})$. In fact, by definition, for every $y \in l^{\infty}(\mathbb{X})$, there is a unique $w \in l^{\infty}(\mathbb{X})$ such that $z_{0} w_{n}-B w_{n}=y_{n}$ for all $n \in \mathbb{Z}$. This shows that given $\tilde{y} \in l^{\infty}(\mathbb{X}) / \mathcal{A}$ there exists a solution $\tilde{w} \in l^{\infty}(\mathbb{X}) / \mathcal{A}$ such that $z_{0} \tilde{w}-\tilde{B} \tilde{w}=\tilde{y}$. Next, we show that such $\tilde{w}$ is unique, that is,

$$
z_{0}(w+\mathcal{A})-B(w+\mathcal{A})=y+\mathcal{A} .
$$

This is obvious from the definition of Condition H. The lemma is proved.

Lemma 2.13. Let $\mathcal{A}$ be the space of all almost automorphic sequences in $\mathbb{X}$, and let $x$ be in $l^{\infty}(\mathbb{X})$ such that $s p_{\mathcal{A}}(x)$ is countable. Moreover, assume that the space $\mathbb{X}$ does not contain any subspace isomorphic to $c_{0}$. Then, $x \in \mathcal{A}$.

Proof. If $s p_{\mathcal{A}}(x)$ is empty, because of the boundedness of the linear operator $\left.\tilde{S}\right|_{\mathcal{M}_{\tilde{x}}}$, the space $\mathcal{M}_{\tilde{x}}$ must be trivial, that is, $\tilde{x}=0$, or in other words, $x$ is almost automorphic.

Suppose that $s p_{\mathcal{A}}(x)$ is not empty. Since $\left.\tilde{S}\right|_{\mathcal{M}_{\tilde{x}}}$ is an isometry in $\mathcal{M}_{\tilde{x}}$ and $s p_{\mathcal{A}}(x)=\sigma\left(\left.\tilde{S}\right|_{\mathcal{M}_{\tilde{x}}}\right)$ is countable, by the Gelfand Theorem there is a point $z_{0}$ in its spectrum that is an eigenvalue of $\left.\tilde{S}\right|_{\mathcal{M}_{\tilde{x}}}$ (for the Gelfand Theorem, see, e.g., [1, 3]). So, we can find an element $\tilde{y} \in \mathcal{M}_{\tilde{x}}$ such that $z_{0} \tilde{y}=\tilde{S} \tilde{y}$. To complete the proof we will show that for each bounded sequence $x \in l^{\infty}(\mathbb{X})$, if $z_{0} x-S x$ is in $\mathcal{A}$, then $x \in \mathcal{A}$.

Let us consider the isomorphism $V_{\lambda}: l^{\infty}(\mathbb{X}) \rightarrow l^{\infty}(\mathbb{X})$ defined as

$$
V_{\lambda} x(n)=\lambda^{n} x_{n}, \forall n \in \mathbb{Z},
$$

where $\lambda \in \Gamma$. Note that $V_{\lambda}$ leaves $\mathcal{A}$ invariant and

$$
V_{\lambda} S V_{\lambda}^{-1}=\lambda^{-1} S \text {. }
$$


Thus, we have

$$
z_{0} x-S x=z_{0}\left(x-z_{0}^{-1} S x\right)=z_{0}\left(x-V_{z_{0}} S V_{z_{0}}^{-1} x\right) .
$$

Therefore, $z_{0} x-S x$ is in $\mathcal{A}$ means that $x-V_{z_{0}} S V_{z_{0}}^{-1} x$ is in $\mathcal{A}$. In turn, this is equivalent to saying that $V_{z_{0}}^{-1} x-S V_{z_{0}}^{-1} x$ is in $\mathcal{A}$ because

$$
x-V_{z_{0}} S V_{z_{0}}^{-1} x=V_{z_{0}}(I-S) V_{z_{0}}^{-1} x
$$

and both $V_{z_{0}}, V_{z_{0}}^{-1}$ are isomorphisms on $\mathcal{A}$. Now we are ready to apply Lemma 2.8 to show that $V_{z_{0}}^{-1} x$ must be almost automorphic, so $x$ must be almost automorphic. (Obviously, if $x$ is almost automorphic, then $\tilde{x}=0$. So, $s p_{\mathcal{A}}(x)$ must be empty.) The lemma is proved.

The main result of this paper will be proved based on the following.

Theorem 2.14. Let $B$ be a bounded linear operator in $\mathbb{X}$ with $\sigma_{\Gamma}(B)$ being countable, and let $\mathbb{X}$ not contain any subspace isomorphic to $c_{0}$. Assume further that $\left\{x_{n}\right\}_{n \in \mathbb{Z}}$ be a bounded sequence that satisfies the equation

$$
x_{n+1}=B x_{n}+y_{n}, \quad n \in \mathbb{Z},
$$

where $\left\{y_{n}\right\}_{n \in \mathbb{Z}}$ is in aa $(\mathbb{X})$. Then $\left\{x_{n}\right\}$ is almost automorphic.

Proof. This theorem is an immediate consequence of Lemmas 2.12 and 2.13 ,

\section{Almost automorphy of Bounded solutions OF EVOLUTION EQUATIONS}

Let us consider equations of the form

$$
\frac{d u(t)}{d t}=A(t) u(t)+f(t), \quad t \in \mathbb{R}
$$

where $f$ is an almost automorphic function with values in $\mathbb{X}$, and $A(t)$ generates a 1-periodic evolutionary process $(U(t, s))_{t \geq s}$ in a Banach space $\mathbb{X}$, that is, a twoparameter family of bounded linear operators that satisfies the following conditions:

i) $U(t, t)=I$ for all $t \in \mathbb{R}$,

ii) $U(t, s) U(s, r)=U(t, r)$ for all $t \geq s \geq r$,

iii) the map $(t, s) \mapsto U(t, s) x$ is continuous for every fixed $x \in \mathbb{X}$,

iv) $U(t+1, s+1)=U(t, s)$ for all $t \geq s$ (1-periodicity),

v) $\|U(t, s)\| \leq N e^{\omega(t-s)}$ for some positive $N, \omega$ independent of $t \geq s$.

We emphasize that the above choice of the period of the equations is merely for the simplification of the notation, but does not mean a restriction. We refer the reader to [1, 7] for more information on the applications of this concept of evolutionary processes to partial differential equations.

An $\mathbb{X}$-valued continuous function $u$ on $\mathbb{R}$ is said to be a mild solution of (3.1) if

$$
u(t)=U(t, s) u(s)+\int_{s}^{t} U(t, \xi) f(\xi) d \xi, \quad \forall t \geq s ; t, s \in \mathbb{R} .
$$

Lemma 3.1. Let $u$ be a bounded mild solution of (3.1) on $\mathbb{R}$ and let $f$ be almost automorphic. Then, $u$ is almost automorphic if and only if the sequence $\{u(n)\}_{n \in \mathbb{Z}}$ is almost automorphic. 
Proof. Necessity: Obviously, if $u$ is almost automorphic, the sequence $\{u(n)\}_{n \in \mathbb{Z}}$ is almost automorphic.

Sufficiency: Let the sequence $\{u(n)\}_{n \in \mathbb{Z}}$ be almost automorphic. We now prove that $u$ is almost automorphic. The proof is divided into several steps:

Step 1: We first suppose that $\left\{n_{k}^{\prime}\right\}$ is a given sequence of integers. Then there exists a subsequence $\left\{n_{k}\right\}$ and a sequence $\{v(n)\}$ such that

$$
\begin{array}{ll}
\lim _{k \rightarrow \infty} u\left(n+n_{k}\right)=v(n) ; & \lim _{k \rightarrow \infty} v\left(n-n_{k}\right)=u(n), \quad \forall n \in \mathbb{Z}, \\
\lim _{k \rightarrow \infty} f\left(t+n_{k}\right)=g(t) ; & \lim _{k \rightarrow \infty} g\left(t-n_{k}\right)=f(t), \quad \forall t \in \mathbb{R} .
\end{array}
$$

For every fixed $t \in \mathbb{R}$, let us denote by $[t]$ the integer part of $t$. Then, define

$$
v(\eta):=U(\eta,[t]) v([t])+\int_{[t]}^{\eta} U(\eta, \xi) g(\xi) d \xi, \quad \eta \in[[t],[t]+1) .
$$

In this way, we can define $v$ on the whole line $\mathbb{R}$. Now we show that

$$
\lim _{k \rightarrow \infty} u\left(t+n_{k}\right)=v(t)
$$

In fact,

$$
\begin{aligned}
\lim _{k \rightarrow \infty}\left\|u\left(t+n_{k}\right)-v(t)\right\| \leq & \lim _{k \rightarrow \infty}\left\|U\left(t+n_{k},[t]+n_{k}\right) u\left([t]+n_{k}\right)-U(t,[t]) v([t])\right\| \\
& +\lim _{k \rightarrow \infty} \int_{[t]}^{t}\|U(t, \eta)\|\left\|f\left(\eta+n_{k}\right)-g(\eta)\right\| d \eta \\
= & \lim _{k \rightarrow \infty}\left\|U(t,[t]) u\left([t]+n_{k}\right)-U(t,[t]) v([t])\right\| \\
& +\lim _{k \rightarrow \infty} \int_{[t]}^{t}\|U(t, \eta)\|\left\|f\left(\eta+n_{k}\right)-g(\eta)\right\| d \eta=0 .
\end{aligned}
$$

Similarly, we can show that

$$
\lim _{k \rightarrow \infty}\left\|v\left(t-n_{k}\right)-u(t)\right\|=0 .
$$

Step 2: Now we consider the general case where $\left\{s_{k}^{\prime}\right\}_{k \in \mathbb{Z}}$ may not be an integer sequence. The main lines are similar to those in Step 1 combined with the strong continuity of the process and the precompactness of the range of the function $f$.

Set $n_{k}^{\prime}=\left[s_{k}^{\prime}\right]$ for every $k$. Since $\left\{t_{k}\right\}_{k \in \mathbb{Z}}$, where $t_{k}:=s_{k}^{\prime}-\left[s_{k}^{\prime}\right]$, is a sequence in $[0,1)$, we can choose a subsequence $\left\{n_{k}\right\}$ from $\left\{n_{k}^{\prime}\right\}$ such that $\lim _{k \rightarrow \infty} t_{k}=t_{0} \in$ $[0,1]$, and (3.3) holds for a function $v$, as shown in Step 1.

Let us first consider the case $0<t_{0}+t-\left[t_{0}+t\right]$. We show that

$$
\lim _{k \rightarrow \infty} u\left(t_{k}+t+n_{k}\right)=\lim _{k \rightarrow \infty} u\left(t_{0}+t+n_{k}\right)=v\left(t_{0}+t\right)
$$

In fact, for sufficiently large $k$, from the above assumption we have $\left[t_{0}+t\right]=\left[t_{k}+t\right]$. Using the 1-periodicity of the process $(U(t, s))_{t \geq s}$ we have

$$
\left\|u\left(t_{k}+t+n_{k}\right)-u\left(t_{0}+t+n_{k}\right)\right\| \leq A(k)+B(k),
$$

where $A(k)$ and $B(k)$ are defined and estimated as below. By the 1-periodicity of the process $(U(t, s))_{t \geq s}$, we have

$$
\begin{aligned}
A(k):= & \| U\left(t_{k}+t+n_{k},\left[t_{k}+t\right]+n_{k}\right) u\left(\left[t_{k}+t\right]+n_{k}\right) \\
& -U\left(t_{0}+t+n_{k},\left[t_{0}+t\right]+n_{k}\right) u\left(\left[t_{0}+t\right]+n_{k}\right) \| \\
= & \left\|U\left(t_{k}+t,\left[t_{0}+t\right]\right) u\left(\left[t_{0}+t\right]+n_{k}\right)-U\left(t_{0}+t,\left[t_{0}+t\right]\right) u\left(\left[t_{0}+t\right]+n_{k}\right)\right\| .
\end{aligned}
$$


Using the strong continuity of the process $(U(t, s))_{t \geq s}$ and the precompactness of the range of the sequence $\{u(n)\}_{n \in \mathbb{Z}}$, we have $\lim _{k \rightarrow \infty} A(k)=0$. Next, we define

$$
B(k):=\left\|\int_{\left[t_{k}+t\right]+n_{k}}^{t_{k}+t+n_{k}} U\left(t_{k}+t+n_{k}, \eta\right) f(\eta) d \eta-\int_{\left[t_{0}+t\right]+n_{k}}^{t_{0}+t+n_{k}} U\left(t_{0}+t+n_{k}, \eta\right) f(\eta) d \eta\right\| .
$$

By the 1-periodicity of the process $(U(t, s))_{t \geq s}$ and $\left[t_{0}+t\right]=\left[t_{k}+t\right]$, we have

$$
\begin{aligned}
B(k)= & \| \int_{0}^{t_{k}+t-\left[t_{k}+t\right]} U\left(t_{k}+t+n_{k},\left[t_{0}+t\right]+n_{k}+\theta\right) f\left(\left[t_{0}+t\right]+n_{k}+\theta\right) d \theta \\
& -\int_{0}^{t_{0}+t-\left[t_{0}+t\right]} U\left(t_{0}+t+n_{k},\left[t_{0}+t\right]+n_{k}+\theta\right) f\left(\left[t_{0}+t\right]+n_{k}+\theta\right) d \theta \| \\
= & \| \int_{0}^{t_{k}+t-\left[t_{0}+t\right]} U\left(t_{k}+t-\left[t_{0}+t\right], \theta\right) f\left(\left[t_{0}+t\right]+n_{k}+\theta\right) d \theta \\
& -\int_{0}^{t_{0}+t-\left[t_{0}+t\right]} U\left(t_{0}+t-\left[t_{0}+t\right], \theta\right) f\left(\left[t_{0}+t\right]+n_{k}+\theta\right) d \theta \| .
\end{aligned}
$$

From the strong continuity of the process $(U(t, s))_{t \geq s}$ and the precompactness of the range of $f$ it follows that $\lim _{k \rightarrow \infty} B(k)=0$. So, in view of Step 1 , we see that (3.5) holds.

Next, we consider the case when $t_{0}+t-\left[t_{0}+t\right]=0$, that is, $t_{0}+t$ is an integer. If $t_{k}+t \geq t_{0}+t$, we can repeat the above argument. So, we omit the details. Now suppose that $t_{k}+t<t_{0}+t$. Then

$$
\left\|u\left(t_{k}+t+n_{k}\right)-u\left(t_{0}+t+n_{k}\right)\right\| \leq C(k)+D(k),
$$

where $C(k)$ and $D(k)$ are defined and estimated as below:

$$
\begin{gathered}
C(k):=\| U\left(t_{k}+t+n_{k},\left[t_{k}+t\right]+n_{k}\right) u\left(\left[t_{k}+t\right]+n_{k}\right) \\
\quad-U\left(t_{0}+t+n_{k}, t_{0}+t-1+n_{k}\right) u\left(t_{0}+t-1+n_{k}\right) \| \\
=\| U\left(t_{k}+t, t_{0}+t-1\right) u\left(t_{0}+t-1+n_{k}\right) \\
\quad-U\left(t_{0}+t, t_{0}+t-1\right) u\left(t_{0}+t-1+n_{k}\right) \| .
\end{gathered}
$$

Now using the strong continuity of the process $(U(t, s))_{t \geq s}$ and the precompactness of the range of the sequence $\{u(n)\}_{n \in \mathbb{Z}}$ we obtain $\lim _{k \rightarrow \infty} C(k)=0$.

As for $D(k)$, we have

$$
\begin{aligned}
D(k):=\| \int_{\left[t_{k}+t\right]+n_{k}}^{t_{k}+t+n_{k}} U\left(t_{k}+t+n_{k}, \eta\right) f(\eta) d \eta \\
\quad-\int_{\left[t_{0}+t\right]+n_{k}-1}^{t_{0}+t+n_{k}} U\left(t_{0}+t+n_{k}, \eta\right) f(\eta) d \eta \| \\
=\| \int_{\left[t_{0}+t\right]+n_{k}-1}^{t_{k}+t+n_{k}} U\left(t_{k}+t+n_{k}, \eta\right) f(\eta) d \eta \\
\quad-\int_{\left[t_{0}+t\right]+n_{k}-1}^{t_{0}+t+n_{k}} U\left(t_{0}+t+n_{k}, \eta\right) f(\eta) d \eta \| \\
=\| \int_{0}^{t_{k}+1-t_{0}} U\left(t_{k}+t, t_{0}+t-1+\theta\right) f\left(t_{0}+t+n_{k}-1+\theta\right) d \theta \\
\quad-\int_{0}^{1} U\left(t_{0}+t, t_{0}+t-1+\theta\right) f\left(t_{0}+t+n_{k}-1+\theta\right) d \theta \| .
\end{aligned}
$$


From the strong continuity of the process $(U(t, s))_{t \geq s}$ and the precompactness of the range of $f$, it follows that $\lim _{k \rightarrow \infty} D(k)=0$. This finishes the proof of the lemma.

As a main result of this paper we have the following theorem.

Theorem 3.2. Let $A(t)$ in (3.1) generate a 1-periodic strongly continuous evolutionary process, and let $f$ be almost automorphic. Assume further that the space $\mathbb{X}$ does not contain any subspace isomorphic to $c_{0}$ and the part of spectrum of the monodromy operator $U(1,0)$ on the unit circle is countable. Then, every bounded mild solution of (3.1) on the real line is almost automorphic.

Proof. The theorem is an immediate consequence of Lemmas 2.12, 2.13, and 3.1. In fact, we need only to prove the sufficiency. Let us consider the discrete equation

$$
u(n+1)=U(n+1, n) u(n)+\int_{n}^{n+1} U(n+1, \xi) f(\xi) d \xi, \quad n \in \mathbb{Z} .
$$

From the 1-periodicity of the process $(U(t, s))_{t \geq s}$, this equation can be re-written in the form

$$
u(n+1)=B u(n)+y_{n}, \quad n \in \mathbb{Z}
$$

where

$$
B:=U(1,0) ; y_{n}:=\int_{n}^{n+1} U(n+1, \xi) f(\xi) d \xi, \quad n \in \mathbb{Z} .
$$

We are going to show that the sequence $\left\{y_{n}\right\}_{n \in \mathbb{Z}}$ defined as above is almost automorphic. In fact, since $f$ is automorphic, for any given sequence $\left\{n_{k}^{\prime}\right\}$ there is a subsequence $\left\{n_{k}\right\}$ and a measurable function $g$ such that $\lim _{k \rightarrow \infty} f\left(t+n_{k}\right)=g(t)$ and $\lim _{m \rightarrow \infty} g\left(t-n_{m}\right)=f(t)$ for every $t \in \mathbb{R}$. Therefore, if we set

$$
w_{n}=\lim _{k \rightarrow \infty} \int_{n+n_{k}}^{n+n_{k}+1} U\left(n+n_{k}, \xi\right) f(\xi) d \xi, \quad n \in \mathbb{Z},
$$

then, by the 1-periodicity of $(U(t, s))_{t \geq s}$ and the Lebesgue Dominated Convergence Theorem, we have

$$
w_{n}=\lim _{k \rightarrow \infty} \int_{n}^{n+1} U(n, \eta) f\left(n_{k}+\eta\right) d \eta=\int_{n}^{n+1} U(n, \eta) g(\eta) d \eta .
$$

Therefore, $\lim _{k \rightarrow \infty} y_{n+n_{k}}=w_{n}$ for every $n \in \mathbb{Z}$. Similarly, we can show that $\lim _{k \rightarrow \infty} w_{n-n_{k}}=y_{n}$.

By Lemma 2.13 since $\{u(n)\}$ is a bounded solution of (3.8), $\mathbb{X}$ does not contain any subspace isomorphic to $c_{0}$, and the part of spectrum of $U(1,0)$ on the unit circle is countable, $\{u(n)\}$ is almost automorphic. By Lemma 3.1 this yields that the solution $u$ itself is almost automorphic.

\section{REFERENCES}

1. W. Arendt, C.J.K. Batty, M. Hieber, F. Neubrander, Vector-valued Laplace transforms and Cauchy problems, Monographs in Mathematics, 96, Birkhäuser Verlag, Basel, 2001. MR 1886588 (2003g:47072)

2. B. Basit, Generalization of two theorems of M. I. Kadets concerning the indefinite integral of abstract almost periodic functions, Mat. Zametki 9 (1971), 311-321. (Russian) MR 0284562 (44:1787)

3. B. Basit, A. J. Pryde, Ergodicity and stability of orbits of unbounded semigroup representations. J. Aust. Math. Soc. 77 (2004), 209-232. MR2083746 (2005g:47073) 
4. A. Berger, S. Siegmund, Y. Yi, On almost automorphic dynamics in symbolic lattices. Ergodic Theory Dynam. Systems 24 (2004), 677-696. MR2060993 (2005b:37018)

5. R. Chill, J. Prüss, Asymptotic behaviour of linear evolutionary integral equations. Integral Equations Operator Theory 39 (2001), 193-213. MR.1817657 (2001m:45015)

6. T. Diagana, G. N'guéŕekata, Nguyen Van Minh, Almost automorphic solutions of evolution equations. Proc. Amer. Math. Soc. 132 (2004), 3289-3298. MR2073304 (2005d:34124)

7. D. Henry, Geometric Theory of Semilinear Parabolic Equations, Lecture Notes in Math., Springer-Verlag, Berlin-New York, 1981. MR0610244 (83j:35084)

8. Y. Hino, S. Murakami, Almost automorphic solutions for abstract functional differential equations. J. Math. Anal. Appl. 286 (2003), 741-752. MR2008862 (2004i:34160)

9. Y. Hino, T. Naito, N.V. Minh, J.S. Shin, Almost Periodic Solutions of Differential Equations in Banach Spaces. Taylor \& Francis, London - New York, 2002. MR.1933682 (2003i:34115)

10. B.M. Levitan, V.V. Zhikov, Almost Periodic Functions and Differential Equations, Moscow Univ. Publ. House, 1978. English translation by Cambridge University Press, 1982. MR0690064 (84g:34004)

11. J. Liu, G. N'Guérékata, Nguyen van Minh, A Massera type theorem for almost automorphic solutions of differential equations. J. Math. Anal. Appl. 299 (2004), no. 2, 587-599. MR.2098262 (2005f:34163)

12. T. Naito, Nguyen Van Minh, R. Miyazaki, Y. Hamaya, Boundedness and almost periodicity dynamical systems, Journal of Difference Equations and Applications 7 (2001), 507-528. MR:1922587 (2003g:39008)

13. G. M. N'Guérékata, Almost automorphic functions and applications to abstract evolution equations, Contemporary Math. 252 (1999), 71-76. MR.1747279(2001a:34097)

14. G. M. N'Guérékata Almost Automorphic and Almost Periodic Functions in Abstract Spaces, Kluwer, Amsterdam, 2001. MR1880351 (2003d:43001)

15. G. M. N'Guérékata, Topics in Almost Automorphy, Springer, New York, 2005. MR2107829 (2005h:34153)

16. W.M. Ruess, Q.P. Vu, Asymptotically almost periodic solutions of evolution equations in Banach spaces, J. Differential Equations 122 (1995), 282-301. MR.1355893 (96i:34143)

17. W. Shen, Y. Yi, Almost Automorphic and Almost Periodic Dynamics in Skew-Product Semiflows, Memoirs of the Amer. Math. Soc. 136 (1998). MR1445493 (99d:34088)

Department of Mathematics, University of West Georgia, Carrollton, Georgia 30118

E-mail address: vnguyen@westga.edu

Department of Mathematics, University of Electro-Communications, Chofu, Tokyo 182-8585, JAPAN

E-mail address: naito@e-one.uec.ac.jp

Department of Mathematics, Morgan State University, 1700 E. Cold Spring Lane, BALTimore, MARYLAND 21251

E-mail address: gnguerek@jewel.morgan.edu 